\title{
Razones para vivir y conducta de salud en estudiantes universitarios con y sin violencia intrafamiliar, según procedencia (pública y privada) de la ciudad de Lima
}

\author{
Reasons for living and health behaviors in university students with and \\ without intrafamily violence, (according to public and private universities) \\ of the city of Lima
}

\author{
Rosa E. Huerta Rosales ${ }^{1}$ \\ Renato Santivañez ${ }^{2}$ \\ Natalia Ramírez ${ }^{3}$ \\ Eliana Delgado ${ }^{4}$ \\ Jessica Ramos ${ }^{5}$ \\ Brenda Oré ${ }^{6}$ \\ Universidad Nacional Mayor de San Marcos
}

Recibido: $11-04-18$

Aceptado: $27-12-18$

\begin{abstract}
Resumen intrafamiliar.

\footnotetext{
${ }^{1}$ Docente. Correspondencia. E-mail: rhuertar@unmsm.edu.pe

${ }^{2}$ Docente. E-mail: rsantivanezo@unmsm.edu.pe

${ }^{3}$ Docente. E-mail: nramirezs@unmsm.edu.pe

${ }^{4}$ Docente. E-mail: edelgadoc@unmsm.edu.pe

${ }^{5}$ Egresada. E-mail: jessicaramosreyes@gmail.com

${ }^{6}$ Egresada. E-mail: bren_ore@hotmail.com
}

Se analizan las variables conductas de salud y razones para vivir, según universidad pública y privada, con y sin violencia intrafamiliar en una muestra de 495 estudiantes, a quienes se les aplicó una ficha de datos y los Cuestionarios Estilos de vida en jóvenes universitarios y Razones para vivir en jóvenes adultos. Existe relación significativa entre las conductas de salud con las razones para vivir. Con violencia, las conductas de salud actividad física y deporte y organización del sueño se asocian con las razones para vivir expectativas futuras, creencias de afrontamiento, autoevaluación positiva y relaciones entre pares; la voluntad personal y los compañeros coadyuvan a enfrentar entornos difíciles. En la pública, actividad física y deporte, organización del descanso y tiempo libre se asocian con expectativas futuras, creencias de afrontamiento, autoevaluación positiva, relaciones entre pares y la familia cuando hay violencia, erigiéndose en conductas protectores. En pública y privada, sin violencia, las relaciones familiares tienen alto significado en lo personal y social. Las mujeres, sin violencia, presentan conductas de salud y razones para vivir significativas no así los hombres. La familia puede ser factor protector o de riesgo en la prevención del suicidio y en el estado de salud.

Palabras clave: Razones para vivir; conducta de salud; estudiante universitario y violencia

(C) Los autores. Este artículo es publicado por la Revista de Investigación en Psicología de la Facultad de Psicología, Universidad Nacional Mayor de San Marcos. Este es un artículo de acceso abierto, distribuido bajo los términos de la licencia Creative Commons Atribucion - No Comercia_Compartir Igual 4.0 Internacional. (http://creativecommons.org/licenses/by-nc-sa/4.0/) que permite el uso no comercial, distribución y reproducción en cualquier medio, siempre que la obra original sea debidamente citada. 


\begin{abstract}
The variables of health behaviors and reasons for living were analyzed, according to public and private universities, with and without intrafamily violence in a sample of 495 students, to whom a data sheet and Lifestyles Questionnaires (CEVJU-r Lima) were applied in university students and Reasons for Living in Young Adults (RFL-YA). There is a significant relationship between health behaviors and reasons for living. Violence, health behaviors, physical activity and sports and sleep organization are associated with future expectations, coping beliefs, positive self-evaluation and peer relations; the personal will and the companions help to face difficult environments. At the public university, physical activity and sports, organization of resting-state activities and free time are associated with future expectations, coping beliefs, positive self-evaluation, peer relations and family when there is violence, becoming protective behaviors. In public and private universities, without violence, family relationships have high personal and social significance. Unlike men, women, without violence, show meaningfull health behaviors and reasons for living. Family can represent a protective or a risk factor in the prevention of suicide and health condition.
\end{abstract}

Keywords: Reasons for living; health behavior; university student and intrafamily violence.

\title{
INTRODUCCIÓN
}

La adolescencia y juventud vienen a ser en la actualidad, etapas vulnerables para desarrollar conductas de riesgo. Uno de los frecuentes problemas que traspone la población a nivel mundial, es el suicidio, considerado como uno de los problema más graves de salud pública por la Organización Mundial de la Salud, destacando que el suicidio es la segunda causa de muerte en las edades de 15 a 29 años, resaltando que el 75 por ciento se produce en países de bajos ingresos (OMS, 2018).

Dentro de los elementos que podrían favorecer la presentación de la conducta suicida, se encuentran los individuales, familiares y sociales (OMS, 2001, citado en Vargas y Saavedra; 2012). En el Perú, Paredes, Orbegoso \& Rosales (2006) reportan que de los usuarios atendidos en un hospital general de Lima entre 1995 a 2004, el mayor número fueron adolescentes y pacientes adultos tempranos, así el 74\% de ellos, presentaban entre 15 y 29 años de edad. Así mismo Vargas, Tovar y Velarde (2010) reportan respecto a la prevalencia y factores asociados con el episodio depresivo en adolescentes de Lima Metropolitana y Callao, 8,6\% de prevalencia actual para el episodio depresivo, la cual se incrementa entre los 15 y 17 años. Frente a esta problemática se hace necesario estudiar los factores protectores y recursos adaptativos que tienen las personas que no se suicidan (Rosero, 2008; Wang, Lightsey, Pietruszka, Uruk, \& Wells, 2007); a lo cual Osman A., Downs, Kopper, Barrios, Baker y Osman, J. (1998) plantean que concentrarse en la valoración de los factores de riesgo suicida no favorece el comprenderlo y prevenirlo en su totalidad; haciéndose relevante estudiar ambos los de riesgo y protectores.

Un factor protector más influyente contra los pensamientos y actos suicidas vienes a ser las razones para vivir, estos se refieren a las afirmaciones que asumen las personas que optan vivir a realizar suicidio cuando afrontan circunstancias de desgracia. Los estudios que han planteado derroteros a seguir frente a esta problemática, son los desarrollados por Linehan, Goodstein, Nielsen y Chiles 
(1983) manifestando que son las creencias y perspectivas vinculadas a la vida las que pueden amortiguar la propensión a la conducta suicida, es decir que las personas con intensas razones para vivir son más propensas a resistir el estrés y la desesperanza y, por ende, a no concretarlo. A diferencia de las personas con carencia de afirmaciones positivas o altas perspectivas por la vida, estas por el contrario poseen pocas ansiedades sobre las consecuencias de su posible suicidio, siendo más proclives a manifestar conductas suicidas. Al estudiar los factores protectores se ha apreciado que la existencia de numerosas razones para vivir se asocia a un menor riesgo y recurrencia para la conducta suicida. Entre las cuales se acentúan, gozar de confianza en uno mismo, poseer habilidades para resistir situaciones de crisis y desazón por la familia (Echávarri, Morales, Bedregal, Barros, Maino y Fischmanb; 2015; García, Palacio, Arias, Ocampo, Calle y Restrepo, 2007).

Por otro lado, cuando el joven se inserta a la vida universitaria tiene que realizar un conjunto de adecuaciones y cambios en sus conductas, las que influyen directamente en su salud, su bienestar y calidad de vida (OMS, 2001). Así la adopción de hábitos no saludables, según el Ministerio de Salud (2007), pueden provocar problemas de salud en una etapa posterior e influir en la esperanza de vida. En la actualidad es alarmante y de intranquilidad, el observar el menoscabo de los hábitos de salud entre los jóvenes peruanos, observándose en los estudios poblacionales un incremento manifiesto de jóvenes que mueren por agresiones, en accidentes de tránsito, uso y abuso de alcohol, fallecimientos por peleas en zonas de diversión por exceso en el consumo de drogas psicoactivas, de embarazos no deseados y de casos con infecciones de transmisión sexual entre ellas el VIH/ SIDA, entre otros (Calle, 2010; Ministerio de Salud). Asimismo, el acrecentamiento conducta sedentaria e inactividad física, de alimentación poco saludable y de fumar, los cuales se configuran como base, con los cardinales factores de riesgo evitables para muchas enfermedades.

Los estilos de vida, que van a determinar la conducta de salud o no, hacen referencia a los modelos de comportamiento de una persona que muestran una relativa permanencia en el tiempo, estos pueden ser influenciados por elementos o variables internas como los motivos, emociones, pensamientos, creencias, habilidades; y externas como las circunstancias socioeconómicas, familiares, sociales, culturales, ecológicas, etc., (Figueroa, 2005; citado en Salazar, Varela, Lema, Tamayo, y Duarte; 2010). La mejora de las conductas de salud tiene que transitar en primer lugar, por la mejora del individuo y de sus competencias y habilidades, así como de la constitución de entornos de interrelación. No se puede pensar por consiguiente, en el desarrollo de conductas saludables sin tener en cuenta el ambiente donde se desenvuelve la persona, especialmente la familia.

La familia es el primer referente para la persona tanto como principal agente de socialización, asimismo de alto significado e involucramiento personal y social, fuente de afecto, dicha, bienestar y sostén, y también puede ser el origen de disgusto, 
incomodidad, estrés y problemas de salud. Las variaciones de la vida familiar inducen la presentación de alteración emocional, inestabilidad y modificaciones en el estado de salud. Estar inserto en desavenencias familiares como violencia intrafamiliar, desencadena respuestas de estrés, entre ellas alteración de la enfermedad crónica o su iniciación, lo cual es grave pues conlleva a construir un sistemas de creencias que afectan en la forma de conducirse, de pensar y tomar decisiones respecto a los acontecimientos que acontecen en el discurrir de la vida (Hederich y Camargo, 2001; citado en Gantiva, Bello, Vanegas y Sastoque, 2010) sobre el que se van a sustentar las razones para vivir. Así la familia, a diferencia de los aprendizajes recibidos que es una fuente de valor, afecto y seguridad, se convierte en el ámbito más que oportuno para manfestarse situaciones de violencia en su interior, Huerta, et al (2015) observaron que la violencia entre los padres que tienen como testigos imperecederos a los niños y adolescentes, tiene que ver con un problema relacional más que estructural.

Violencia familiar, se refiere a cualesquiera de las formas de violencia que se presentan en las relaciones entre los miembros de una familia. Se designa como relación de abuso a la manera en que se interrelacionan, la cual, insertada en un ambiente de desequilibrio de poder, subsume conductas de una de las partes que, por acción u omisión, producen perjuicio físico y/o psicológico a otro integrante de la relación (Corsi, 1997). En nuestro país, el estudio realizado en el año 2009 por el Ministerio de Salud, denominado Análisis de Situación de Salud de las y los Adolescentes, detectó que los conflictos psicosociales por los cuales las y los adolescentes pretenden suicidarse, son similares en población de las diferentes regiones del país, es decir tanto para la costa, sierra y selva, apreciándose como primer motivo aquellos vinculados con las desavenencia con los padres. Es así que la familia, considerada como principal factor protector, puede también llegar a ser un factor de riesgo cuando surgen discordias al interior de ella.

Para el estudio se planeó como objetivos, analizar la relación entre los factores de razones para vivir con las dimensiones de la conducta de salud en estudiantes universitarios con y sin violencia intrafamiliar según procedencia (pública y privada) de la ciudad de Lima. Al mismo tiempo comprobar las diferencias entre los factores de razones para vivir en los estudiantes universitarios con y sin violencia familiar con las dimensiones de la conducta de salud según procedencia (pública y privada) y según género.

\section{MÉTODO}

El estudio es de tipo sustantivo, que establece procedimientos dentro del método correlacional pues permitió conocer tanto la intensidad como la dirección de la relación entre las razones para vivir y las conductas de salud en estudiantes universitarios con y sin violencia intrafamiliar, según procedencia (pública y privada) 
de la ciudad de Lima. El diseño fue descriptivo correlacional, transversal, los datos se recolectaron en un solo momento (Hernández, Fernández y Baptista, 2010).

\section{Muestra}

La población de la investigación está conformado por estudiantes universitarios de sexto a noveno ciclo que asisten a universidades públicas y privadas de la ciudad de Lima. La selección de la muestra se determinó aplicándose un muestreo no probabilístico e intencional.

La muestra quedo establecida por 495 estudiantes universitarios de ambos sexos que se organizaron según edad, sexo, procedencia de universidad pública y privada, siendo el grupo de estudiantes de universidad pública el 64.2 por ciento (318) y privada el 35.8 por ciento (177); en cuanto al género masculino fueron 225 y femenino 270; asimismo en la muestra total el $59.6 \%$ estudia en el turno diurno y el $40.4 \%$ en el turno nocturno.

Las edades de la muestra, oscilan entre 19 y 59 años prevaleciendo la edad de 19 a 22 años tanto en el grupo de estudiantes de universidad pública (58.8\%,) como en la privada (60.5), siendo las edades de 26 a 59 años las menores (8.5\%). En lo referente a la presencia de violencia intrafamiliar se aprecia que en el caso de quienes proceden de universidades públicas el $59.7 \%$ ha recibido violencia, mientras que el $40.3 \%$ no la ha recibido. Con respecto a la presentación de violencia en el grupo de las universidades privadas el $52.0 \%$ no la ha recibido, mientras que el $48.0 \%$ sí la ha recibido.

\section{Variables de Estudio}

Las variables de estudio corresponden a los indicadores del Inventario Razones para Vivir, versión para jóvenes adultos (RFL-YA), compuesta por cinco factores; y el Cuestionario de Estilos de Vida en Jóvenes Universitarios (CEVJU-r Lima), compuesta por seis dimensiones; las que fueron aplicadas en dos grupos (con y sin violencia intrafamiliar) asimismo se consideró la procedencia pública y privada.

\section{Técnicas e instrumentos de recolección de datos.}

Para la recolección de datos se establecieron las universidades públicas y privadas así como las carreras, tomándose para ello ciencias de la salud, ciencias económicas e ingenierías, de la ciudad de Lima. Los instrumentos fueron aplicados de manera grupal.

Se utilizó la técnica de la encuesta y dos instrumentos. Se revisaron los instrumentos a ser aplicados, ambos cuestionarios se encuentran adaptados y validados a población de universitarios, además se aplicó una ficha de datos sociodemográficos elaborado por el investigador para recoger las edades, presencia o no de violencia intrafamiliar, así como para distinguir la procedencia de la universidad. 


\section{Instrumentos:}

Ficha de datos sociodemográficos: Construida por el investigador. Su aplicación se realizó de forma colectiva. Permite recoger características relevantes de los individuos estudiados como, tipo de universidad de origen, edad, sexo, existencia o no violencia.

Cuestionario de Estilos de Vida en Jóvenes Universitarios (CEVJU-r Lima) elaborado por Salazar, Valera, Lema, Tamayo, y Duarte (2009) y adaptado en Perú por Chau y Saravia (2014). Presenta validez de contenido y validez de constructo, apreciándose un Kayser Mayer Olkin (KMO) puntaciones en sus seis dimensiones o áreas entre 0,62 a $0,82(\mathrm{p}<0,01)$, asimismo en el análisis factorial se apreciaron los seis factores. Con respecto a la confiabilidad obtiene un Alpha de Cronbach en las 6 áreas entre 0.64 a 0.80. Es un instrumento de auto informe, consta de 30 ítems, su aplicación es en sujetos de 16 a 24 años, las respuestas es en una escala tipo Likert. Evalúa 6 dimensiones de las prácticas del estilo de vida que conllevan a evaluar la presentación de conductas de salud: Actividad física y deporte, Organización del descanso o tiempo libre, Autocuidado y cuidado médico, Hábitos alimentarios, Consumo de alcohol, tabaco y otras drogas, Organización del sueño. Para el presente estudió de manera similar presenta validez de contenido y validez de constructo, así como un coeficiente de consistencia interna Alpha de Cronbach de 0.766 en la prueba total, y para las 6 dimensiones ésta oscila de 0.67 a 0.84 , que indica que el cuestionario mide dentro de los márgenes de error permitido y los ítems presentan validez de construcción.

Razones para vivir en jóvenes adultos (RFL-YA), elaborada por Linehan, et al., (1983), adaptada y validada en Perú por Cassaretto y Martinez (2012). Presenta validez de contenido y validez de constructo alcanzando un KMO de .91 $(\mathrm{p}<001)$, una estructura factorial consistente pues todos los ítems se situaron en sus factores que le pertenecen. En cuanto a la confiabilidad alcanza un Alpha de Cronbach para la escala total de 0.93 y para los factores entre 0.64 y 0.80 . Consta de 32 ítems, su aplicación en sujetos de 16 a 24 años, presenta 5 factores: Expectativas futuras, Creencias de afrontamiento, Autoevaluación positiva, Relaciones entre pares, Relaciones familiares. Para el estudio se encontró un Alpha de Cronbach de 0.948 en el cuestionario total, y en sus 5 factores fluctúan de 0.819 a 0.90 , que indican consistencia interna y que el cuestionario mide dentro de los márgenes de error permitidos.

\section{RESULTADOS}

1. Relación entre los factores de las razones para vivir con las dimensiones de la conducta de salud en estudiantes universitarios con y sin violencia intrafamiliar según procedencia (pública y privada) de la ciudad de Lima. Los datos obtenidos de ambas variables se correlacionaron con el coeficiente 
Rho de Spearman hallando relaciones significativas y positivas entre las dimensiones de la conducta de salud con los factores de las razones para vivir:

- Análisis sin considerar la presencia de violencia intrafamiliar. Este análisis se hace relevante pues nos permite observar la situación del estudiante universitario respecto a sus conductas de salud y razones para vivir; así se observa que:

Existe correlación significativa, positiva y baja entre la conducta de salud actividad física y deporte con las razones para vivir autoevaluación positiva y relaciones entre pares. Relación similar se encuentra entre la conducta de salud organización del descanso y tiempo libre con las razones para vivir expectativas futuras, creencias de afrontamiento, autoevaluación positiva, relaciones entre pares y relaciones familiares. También Existe correlación significativa, positiva y baja entre el autocuidado y cuidado médico con las razones para vivir creencias de afrontamiento, autoevaluación positiva y relaciones entre pares. Asimismo los hábitos alimentarios se asociación con las razones para vivir relaciones entre pares y relaciones familiares, es decir con razones de su ámbito personal y social; en cuanto a la conducta de salud se aprecia cuando existe bajo consumo de alcohol, tabaco y otras drogas se asocia con la razón para vivir relaciones familiares, al cual le otorga importancia. Por otro lado la conducta organización del sueño se asocia con las razones para vivir expectativas futuras, creencias de afrontamiento, autoevaluación positiva y relaciones familiares.

- Cuando se examinan los datos, en la muestra general, respecto a la correlación entre conductas de salud y razones para vivir según presencia de violencia intrafamiliar se halla que:

Existe correlación estadísticamente significativa, positiva y baja en el grupo con violencia entre la conducta de salud actividad física y deporte con la razón para vivir expectativas futuras, creencias de afrontamiento, autoevaluación positiva y relaciones entre pares. Igualmente entre hábitos alimentarios con la razón para vivir relaciones familiares; y en entre la conducta de salud consumo de alcohol y otras drogas con relaciones entre pares; así como también en la conducta organización del sueño con las razones para vivir expectativas futuras, autoevaluación positiva, creencias de afrontamiento, relaciones entre pares y familiares.

En el grupo sin violencia existe asociación significativa, positiva y baja entre la conducta autocuidado y cuidado médico con las razones para vivir autoevaluación positiva y relaciones entre pares; asimismo entre la conducta uso de alcohol, tabaco y otras drogas con las razones 
expectativas futuras, autoevaluación positiva, creencias de afrontamiento y relaciones familiares, así como también entre la conducta organización del sueño con la razón autoevaluación positiva.

Referente a la conducta de salud organización del descanso o tiempo existe asociación significativa, positiva y baja entre con las razones para vivir expectativas futuras, creencias de afrontamiento, autoevaluación positiva, relaciones entre pares y familiares, se observa en ambos grupos con y sin violencia. Ello nos permite afirmar que las conductas de salud intervienen en las razones para vivir y viceversa, influenciada por la presentación o no de violencia intrafamiliar. Tal como se observa en la Tabla 1.

Tabla 1

Correlación entre conductas de salud y razones para vivir según presencia de violencia, muestra total

\begin{tabular}{|c|c|c|c|c|c|c|c|c|c|c|c|}
\hline & & \multicolumn{2}{|c|}{$\begin{array}{l}\text { Expectativas } \\
\text { futuras }\end{array}$} & \multicolumn{2}{|c|}{$\begin{array}{l}\text { Creencias de } \\
\text { afrontamiento }\end{array}$} & \multicolumn{2}{|c|}{$\begin{array}{l}\text { Autoevaluación } \\
\text { positiva }\end{array}$} & \multicolumn{2}{|c|}{$\begin{array}{l}\text { Relaciones } \\
\text { entre pares }\end{array}$} & \multicolumn{2}{|c|}{$\begin{array}{l}\text { Relaciones } \\
\text { familiares }\end{array}$} \\
\hline & & $\mathrm{C} / \mathrm{VIF}$ & $\mathrm{S} / \mathrm{VIF}$ & $\mathrm{C} / \mathrm{VIF}$ & $\mathrm{S} / \mathrm{VIF}$ & $\mathrm{C} / \mathrm{VIF}$ & $\mathrm{S} / \mathrm{VIF}$ & $\mathrm{C} / \mathrm{VIF}$ & $\mathrm{S} / \mathrm{VIF}$ & $\mathrm{C} / \mathrm{VIF}$ & $\mathrm{S} / \mathrm{VIF}$ \\
\hline \multirow{3}{*}{ Actividad física y deporte } & Rho &, $\mathbf{1 2 8}^{*}$ & .010 &, $\mathbf{1 3 5}^{*}$ & .002 &, $\mathbf{1 2 8}^{*}$ & .071 &, $137^{k}$ & .082 & .055 & -.039 \\
\hline & $\mathrm{p}$ & .034 & .888 & .025 & .977 & .033 & .297 & .023 & .227 & .361 & .563 \\
\hline & $\mathrm{N}$ & 275 & 220 & 275 & 220 & 275 & 220 & 275 & 220 & 275 & 220 \\
\hline \multirow{3}{*}{ Organización del descanso } & Rho &, $218^{* *}$ &, $170^{*}$ &, $192^{* *}$ &, $165^{*}$ &, $251^{* *}$ &, $151^{*}$ &, $205^{* *}$ &, $165^{*}$ &, $185^{* *}$ & $243^{* *}$ \\
\hline & $\mathrm{p}$ & .000 & .012 & .001 & .014 & .000 & .025 & .001 & .014 & .002 & .000 \\
\hline & $\mathrm{N}$ & 275 & 220 & 275 & 220 & 275 & 220 & 275 & 220 & 275 & 220 \\
\hline \multirow{3}{*}{$\begin{array}{l}\text { Autocuidado y cuidado } \\
\text { médico }\end{array}$} & Rho & .027 & .115 & .108 & .097 & .104 &, $\mathbf{1 7 0}^{*}$ & .031 &, $169^{*}$ & .012 & .106 \\
\hline & $\mathrm{p}$ & .661 & .088 & .073 & .153 & .085 & .011 & .605 & .012 & .844 & .116 \\
\hline & $\mathrm{N}$ & 275 & 220 & 275 & 220 & 275 & 220 & 275 & 220 & 275 & 220 \\
\hline \multirow{3}{*}{ Hábitos alimentarios } & Rho & -.084 & .028 & -.041 & .049 & -.029 & .040 & -.103 & -.117 &,$- 138^{*}$ & -.035 \\
\hline & $\mathrm{p}$ & .163 & .679 & .501 & .466 & .630 & .559 & .087 & .083 & .022 & .610 \\
\hline & $\mathrm{N}$ & 275 & 220 & 275 & 220 & 275 & 220 & 275 & 220 & 275 & 220 \\
\hline \multirow{3}{*}{$\begin{array}{l}\text { Consumo de alcohol, } \\
\text { tabaco y otras drogas }\end{array}$} & Rho & .003 &, $300^{* *}$ & .002 & ,204** & .003 & ,209 &,$- 145^{*}$ & .127 & .028 &, $226^{* *}$ \\
\hline & $\mathrm{p}$ & .955 & .000 & .971 & .002 & .956 & .002 & .016 & .060 & .643 & .001 \\
\hline & $\mathrm{N}$ & 275 & 220 & 275 & 220 & 275 & 220 & 275 & 220 & 275 & 220 \\
\hline \multirow{3}{*}{ Organización del sueño } & Rho &, $139^{*}$ & .112 &, $122^{*}$ & .091 &, $\mathbf{1 4 3}^{*}$ &, $\mathbf{1 4 3}^{*}$ &, $119^{*}$ & .000 &, $146^{*}$ & .112 \\
\hline & $\mathrm{p}$ & .021 & .096 & .044 & .180 & .018 & .033 & .049 & .997 & .015 & .098 \\
\hline & $\mathrm{N}$ & 275 & 220 & 275 & 220 & 275 & 220 & 275 & 220 & 275 & 220 \\
\hline
\end{tabular}

**. La correlación es significativa en el nivel 0,01 (bilateral).

*. La correlación es significativa en el nivel 0,05 (bilateral).

- Respecto a la correlación entre conductas de salud y razones para vivir según presencia de violencia en universidades Públicas, se encuentra que:

El análisis en el grupo con violencia se aprecia que existe correlación significativa, positiva y baja entre la conducta de salud actividad física y deporte con las razones o afirmaciones para vivir expectativas 
futuras, creencias de afrontamiento, autoevaluación positiva, relaciones entre pares y relaciones familiares; correlación similar se da entre las conductas de salud organización del tiempo libre y del sueño con las afirmaciones para vivir expectativas futuras, creencias de afrontamiento, autoevaluación positiva, relaciones entre pares y familiares. En este grupo se puede apreciar que las conductas de salud se relacionan con las razones para vivir, lo cual son manifestaciones de factores resilientes a pesar de vivir en familias con violencia intrafamiliar. Asimismo en este grupo con violencia se da asociación significativa y negativa entre hábitos alimentarios con relaciones familiares, es decir que el entorno familiar se hace relevante para la conducta de salud.

Referente al grupo sin violencia, existe correlación estadísticamente significativa, positiva y baja entre las conductas actividad física y deporte, autocuidado y cuidado médico, organización del sueño y hábitos alimentarios con la razón para vivir autoevaluación positiva. Igualmente en este grupo existe asociación positiva entre organización del descanso o tiempo libre con relaciones familiares; así como también entre el bajo consumo de alcohol, tabaco y otras drogas con creencias de afrontamiento. Tabla 2.

Tabla 2

Correlación entre conductas de salud y razones para vivir según presencia de violencia en universidades públicas

\begin{tabular}{|c|c|c|c|c|c|c|c|c|c|c|c|}
\hline & & \multicolumn{2}{|c|}{$\begin{array}{l}\text { Expectativas } \\
\text { futuras }\end{array}$} & \multicolumn{2}{|c|}{$\begin{array}{l}\text { Creencias de } \\
\text { afrontamiento }\end{array}$} & \multicolumn{2}{|c|}{$\begin{array}{c}\text { Autoevaluación } \\
\text { positiva }\end{array}$} & \multicolumn{2}{|c|}{$\begin{array}{l}\text { Relaciones } \\
\text { entre pares }\end{array}$} & \multicolumn{2}{|c|}{$\begin{array}{l}\text { Relaciones } \\
\text { familiares }\end{array}$} \\
\hline & & $\mathrm{C} / \mathrm{VIF}$ & S/VIF & $\mathrm{C} / \mathrm{VIF}$ & S/VIF & $\mathrm{C} / \mathrm{VIF}$ & S/VIF & $\mathrm{C} / \mathrm{VIF}$ & S/VIF & $\mathrm{C} / \mathrm{VIF}$ & S/VIF \\
\hline \multirow{3}{*}{$\begin{array}{l}\text { Actividad } \\
\text { física y } \\
\text { deporte }\end{array}$} & Rho &, $205^{* *}$ & .137 &, $\mathbf{1 8 5}^{*}$ & .064 &, $210^{k *}$ &, $207^{*}$ &, $235^{* *}$ & .102 &, $145^{*}$ & -.077 \\
\hline & $\mathrm{p}$ & .005 & .124 & .011 & .473 & .004 & .019 & .001 & .252 & .047 & .390 \\
\hline & $\mathrm{N}$ & 190 & 128 & 190 & 128 & 190 & 128 & 190 & 128 & 190 & 128 \\
\hline \multirow{3}{*}{$\begin{array}{l}\text { Organización } \\
\text { del descanso } \\
\text { o tiempo libre }\end{array}$} & Rho &, $225^{k *}$ & .152 &, $265^{k *}$ & .160 &, $282^{k *}$ & .082 &, $190^{k * k}$ & .118 &, $221^{k * k}$ &, $\mathbf{2 3 0}^{k *}$ \\
\hline & $\mathrm{p}$ & .002 & .086 & .000 & .071 & .000 & .358 & .009 & .184 & .002 & .009 \\
\hline & $\mathrm{N}$ & 190 & 128 & 190 & 128 & 190 & 128 & 190 & 128 & 190 & 128 \\
\hline \multirow{3}{*}{$\begin{array}{l}\text { Autocuidado } \\
\text { y cuidado } \\
\text { médico }\end{array}$} & Rho & .070 & .113 & .131 & .129 &, $162^{*}$ &, $197^{\star}$ & .109 & .071 & .043 & .161 \\
\hline & $\mathrm{p}$ & .339 & .203 & .071 & .146 & .025 & .026 & .134 & .423 & .559 & .069 \\
\hline & $\mathrm{N}$ & 190 & 128 & 190 & 128 & 190 & 128 & 190 & 128 & 190 & 128 \\
\hline \multirow{3}{*}{$\begin{array}{l}\text { Hábitos } \\
\text { alimentarios }\end{array}$} & Rho & -.100 & .064 & -.066 & .039 & -.109 &, $206^{*}$ & -.131 & -.011 &,$- 167^{*}$ & .078 \\
\hline & $\mathrm{p}$ & .168 & .471 & .364 & .661 & .136 & .020 & .072 & .903 & .022 & .380 \\
\hline & $\mathrm{N}$ & 190 & 128 & 190 & 128 & 190 & 128 & 190 & 128 & 190 & 128 \\
\hline \multirow{3}{*}{$\begin{array}{l}\text { Consumo } \\
\text { de alcohol, } \\
\text { tabaco y otras } \\
\text { drogas }\end{array}$} & Rho & -.058 & .131 & -.024 &, $206^{k}$ & .041 & .093 & -.138 & .067 & .055 & .143 \\
\hline & $\mathrm{p}$ & .424 & .141 & .743 & .020 & .575 & .297 & .057 & .455 & .450 & .108 \\
\hline & $\mathrm{N}$ & 190 & 128 & 190 & 128 & 190 & 128 & 190 & 128 & 190 & 128 \\
\hline \multirow{3}{*}{$\begin{array}{l}\text { Organización } \\
\text { del sueño }\end{array}$} & Rho &, $167^{*}$ & .115 &, $158^{*}$ & .134 & .109 &, $\mathbf{3 4 3}^{\text {k*t }}$ & .080 & .078 & .122 & .150 \\
\hline & $\mathrm{p}$ & .021 & .196 & .030 & .130 & .133 & .000 & .273 & .383 & .094 & .092 \\
\hline & $\mathrm{N}$ & 190 & 128 & 190 & 128 & 190 & 128 & 190 & 128 & 190 & 128 \\
\hline
\end{tabular}

**. La correlación es significativa en el nivel 0,01 (bilateral).

*. La correlación es significativa en el nivel 0,05 (bilateral). 
- Respecto a la correlación entre conductas de salud y razones para vivir según presencia de violencia en universidades Privadas:

En este grupo cuando existe violencia, se aprecia correlación significativa, positiva y baja entre organización del descanso o tiempo libre con expectativas futuras, autoevaluación positiva y relaciones entre pares.

En lo referente al grupo sin violencia, igualmente se aprecia relación significativa y positiva entre organización del descanso o tiempo libre con expectativas futuras, creencias de afrontamiento, relaciones entre pares y familiares. De manera similar se observa entre autocuidado y cuidado médico con relaciones entre pares y; entre consumo de alcohol, tabaco y otras drogas con relaciones familiares. Ello nos permite observar que las conductas de salud se desarrollan en ambientes familiares con ausencia de violencia intrafamiliar, lo que favorece la construcción de razones para vivir y que la conducta de bajo consumo de alcohol se relaciona de manera importante con un entorno familiar funcional. Como se considera en la Tabla 3.

\section{Tabla 3}

Correlación entre conductas de salud y razones para vivir según presencia de violencia en universidades privadas

\begin{tabular}{|c|c|c|c|c|c|c|c|c|c|c|c|}
\hline & & \multicolumn{2}{|c|}{$\begin{array}{l}\text { Expectativas } \\
\text { futuras }\end{array}$} & \multicolumn{2}{|c|}{$\begin{array}{l}\text { Creencias de } \\
\text { afrontamiento }\end{array}$} & \multicolumn{2}{|c|}{$\begin{array}{c}\text { Autoevaluación } \\
\text { positiva }\end{array}$} & \multicolumn{2}{|c|}{$\begin{array}{l}\text { Relaciones } \\
\text { entre pares }\end{array}$} & \multicolumn{2}{|c|}{$\begin{array}{l}\text { Relaciones } \\
\text { familiares }\end{array}$} \\
\hline & & $\mathrm{C} / \mathrm{VIF}$ & $\mathrm{S} / \mathrm{VIF}$ & $\mathrm{C} / \mathrm{VIF}$ & $\mathrm{S} / \mathrm{VIF}$ & $\mathrm{C} / \mathrm{VIF}$ & $\mathrm{S} / \mathrm{VIF}$ & $\mathrm{C} / \mathrm{VIF}$ & $\mathrm{S} / \mathrm{VIF}$ & $\mathrm{C} / \mathrm{VIF}$ & $\mathrm{S} / \mathrm{VIF}$ \\
\hline \multirow{3}{*}{$\begin{array}{l}\text { Actividad } \\
\text { física y deporte }\end{array}$} & Rho & .068 & -.170 & .079 & .001 & .099 & -.047 & -.055 & .012 & -.120 & -.149 \\
\hline & $\mathrm{p}$ & .538 & .105 & .473 & .995 & .369 & .658 & .615 & .909 & .274 & .155 \\
\hline & $\mathrm{N}$ & 85 & 92 & 85 & 92 & 85 & 92 & 85 & 92 & 85 & 92 \\
\hline \multirow{3}{*}{$\begin{array}{l}\text { Organización } \\
\text { del descanso o } \\
\text { tiempo libre }\end{array}$} & Rho &, $279^{* * * *}$ &, $280^{\text {t** }}$ & .209 &, $258^{\star}$ &, $285^{* *}$ & .134 &, $266^{*}$ &, $228^{*}$ & .099 &, $246^{*}$ \\
\hline & $\mathrm{p}$ & .010 & .007 & .054 & .013 & .008 & .203 & .014 & .029 & .368 & .018 \\
\hline & $\mathrm{N}$ & 85 & 92 & 85 & 92 & 85 & 92 & 85 & 92 & 85 & 92 \\
\hline \multirow{3}{*}{$\begin{array}{l}\text { Autocuidado } \\
\text { y cuidado } \\
\text { médico }\end{array}$} & Rho & .025 & .183 & .166 & .195 & .135 & .180 & -.136 &, $\mathbf{2 5 3}^{*}$ & -.056 & .124 \\
\hline & $\mathrm{p}$ & .818 & .081 & .129 & .063 & .217 & .086 & .214 & .015 & .613 & .239 \\
\hline & $\mathrm{N}$ & 85 & 92 & 85 & 92 & 85 & 92 & 85 & 92 & 85 & 92 \\
\hline \multirow{3}{*}{$\begin{array}{l}\text { Hábitos } \\
\text { alimentarios }\end{array}$} & Rho & -.127 & .005 & -.064 & .047 & .042 & -.025 & -.203 & -.059 & -.200 & -.058 \\
\hline & $\mathrm{p}$ & .248 & .965 & .558 & .657 & .705 & .816 & .062 & .576 & .067 & .581 \\
\hline & $\mathrm{N}$ & 85 & 92 & 85 & 92 & 85 & 92 & 85 & 92 & 85 & 92 \\
\hline \multirow{3}{*}{$\begin{array}{l}\text { Consumo de } \\
\text { alcohol, tabaco } \\
\text { y otras drogas }\end{array}$} & Rho & -.048 & .191 & .006 & .089 & -.136 & .109 & -.213 & .150 & -.033 &, $294^{\text {** }}$ \\
\hline & $\mathrm{p}$ & .665 & .069 & .960 & .397 & .214 & .299 & .051 & .155 & .761 & .004 \\
\hline & $\mathrm{N}$ & 85 & 92 & 85 & 92 & 85 & 92 & 85 & 92 & 85 & 92 \\
\hline \multirow{3}{*}{$\begin{array}{l}\text { Organización } \\
\text { del sueño }\end{array}$} & Rho & .004 & .204 & .146 & .043 & .085 & .037 & .078 & .012 & .122 & .146 \\
\hline & $\mathrm{p}$ & .971 & .051 & .181 & .684 & .439 & .728 & .479 & .912 & .268 & .166 \\
\hline & $\mathrm{N}$ & 85 & 92 & 85 & 92 & 85 & 92 & 85 & 92 & 85 & 92 \\
\hline
\end{tabular}

*. La correlación es significativa en el nivel 0,05 (bilateral).

**. La correlación es significativa en el nivel 0,01 (bilateral). 
2. Comprobar las diferencias entre los factores de razones para vivir en los estudiantes universitarios con y sin violencia familiar con las dimensiones de la conducta de salud según procedencia (pública y privada) de la ciudad de Lima. Para el este análisis, se ha diferenciado ambos grupos, utilizando el análisis U de Mann Whitney, con el objeto de conocer si difieren en función a la presentación o no de violencia intrafamiliar, así en la muestra total se encuentra:

En la Tabla 4 se observa, en la muestra total según violencia, diferencia significativa en el grupo sin violencia en las conductas organización del descanso (264.33; $p=0.021$ ), autocuidado y cuidado médico (267.32; $p=0.007$ ), organización del sueño (267.03; $\mathrm{p}=0.008)$; en comparación con el grupo con violencia (234.93), (232.55) y (232.78) como se aprecia en la diferencia del rango medio respectivamente.

En lo que respecta a las razones para vivir, igualmente solo se aprecian diferencias significativas en el grupo sin violencia en relaciones familiares (274.38; $\mathrm{p}=0.000)$, en comparación al grupo con violencia (226.90).

Ello nos permite afirmar que la ausencia de violencia intrafamiliar favorecen las conductas de salud y que la familia es relevante como razón para vivir.

\section{Tabla 4}

Comparación de los factores de las razones para vivir y las dimensiones de la conducta de salud según violencia intrafamiliar en la muestra total

\begin{tabular}{|c|c|c|c|c|}
\hline Variable & $\begin{array}{c}\text { U de Mann- } \\
\text { Whitney }\end{array}$ & $\begin{array}{c}\text { Rango promedio } \\
\text { C/VIF } \\
(\mathrm{N}=275)\end{array}$ & $\begin{array}{c}\text { Rango promedio } \\
\text { S/VIF } \\
(\mathrm{N}=220)\end{array}$ & $\mathrm{p}$ \\
\hline Actividad física y deporte & 29828.000 & 246.47 & 249.92 & .787 \\
\hline Organización del descanso o tiempo libre & 26657.000 & 234.93 & 264.33 & .021 \\
\hline Autocuidado y cuidado médico & 26000.000 & 232.55 & 267.32 & .007 \\
\hline Hábitos alimentarios & 27674.500 & 257.37 & 236.29 & .102 \\
\hline Consumo de alcohol, tabaco y otras drogas & 29406.500 & 251.07 & 244.17 & .570 \\
\hline Organización del sueño & 26063.500 & 232.78 & 267.03 & .008 \\
\hline Expectativas futuras & 29438.500 & 250.95 & 244.31 & .607 \\
\hline Creencias de afrontamiento & 29475.500 & 250.82 & 244.48 & .624 \\
\hline Autoevaluación positiva & 28061.000 & 240.04 & 257.95 & .165 \\
\hline Relaciones entre pares & 27468.500 & 237.89 & 260.64 & .078 \\
\hline Relaciones familiares & 24446.500 & 226.90 & 274.38 & .000 \\
\hline
\end{tabular}


3. Comparar los factores de razones para vivir en estudiantes universitarios con y sin violencia intrafamiliar, según procedencia (pública y privada) de la ciudad de Lima

Para la comparación, se ha contrastado ambos grupos, utilizando el análisis U de Mann Whitney, a fin de saber si divergen en función a la presencia o no de violencia intrafamiliar.

Como se observa en la Tabla 5, según los valores $\mathrm{p}$, existe una diferencia estadísticamente significativa en el grupo sin violencia intrafamiliar, tanto en los de universidad pública $(\mathrm{p}=0.025)$ alcanzando un rango promedio de 173.58; como en los de universidad privada $(\mathrm{p}=0.002)$ con un rango promedio de 100.18, en la razón para vivir de relaciones familiares. Lo cual permite destacar, una vez más, que el grupo familiar tiene gran relevancia para el estudiante universitario como fuente de soporte personal y social. Asimismo se observa, en los de universidad privada, que los rangos medios más altos son en el grupo sin violencia en las razones para vivir expectativas futuras, autoevaluación positiva y relación entre pares. Con respecto a los de universidad pública se encuentran, en el grupo sin violencia las razones para vivir autoevaluación positiva y relaciones entre pares y con violencia en la razón para vivir expectativas futuras, lo cual indicaría la afectación del universitario respecto a sus planes y metas futuras, cuando existe discordia familiar.

Tabla 5

Comparación de las razones para vivir según violencia intrafamiliar en universidades públicas y privadas

\begin{tabular}{|c|c|c|c|c|c|c|c|c|}
\hline \multirow[b]{2}{*}{ Razones para vivir } & \multicolumn{4}{|c|}{ Pública } & \multicolumn{4}{|c|}{ Privada } \\
\hline & $\begin{array}{c}\text { Ude } \\
\text { Mann- } \\
\text { Whitney }\end{array}$ & $\begin{array}{c}\text { Rango } \\
\text { promedio } \\
\mathrm{C} / \mathrm{VIF} \\
(\mathrm{N}=275)\end{array}$ & $\begin{array}{c}\text { Rango } \\
\text { promedio } \\
\text { S/VIF } \\
(\mathrm{N}=220)\end{array}$ & $\mathrm{p}$ & $\begin{array}{c}\text { Ude } \\
\text { Mann- } \\
\text { Whitney }\end{array}$ & $\begin{array}{c}\text { Rango } \\
\text { promedio } \\
\text { C/VIF } \\
(\mathrm{N}=275)\end{array}$ & $\begin{array}{c}\text { Rango } \\
\text { promedio } \\
\mathrm{S} / \mathrm{VIF} \\
(\mathrm{N}=220)\end{array}$ & $\mathrm{p}$ \\
\hline Expectativas futuras & 11211.500 & 164.49 & 152.09 & .237 & 3640.500 & 85.83 & 91.93 & .428 \\
\hline Creencias de afrontamiento & 11989.500 & 160.40 & 158.17 & .832 & 3757.000 & 90.80 & 87.34 & .653 \\
\hline Autoevaluación positiva & 11978.000 & 158.54 & 160.92 & .820 & 3300.000 & 81.82 & 95.63 & .072 \\
\hline Relaciones entre pares & 11490.500 & 155.98 & 164.73 & .404 & 3439.500 & 83.46 & 94.11 & .166 \\
\hline Relaciones familiares & 10357.500 & 150.01 & 173.58 & .025 & 2881.500 & 76.90 & 100.18 & .002 \\
\hline
\end{tabular}

4. Comparar las dimensiones de la conducta de salud en estudiantes universitarios con y sin violencia intrafamiliar, según procedencia (pública y privada) de la ciudad de Lima.

En la tabla 6 se observan en las dimensiones de la conducta de salud, según los valores p que existe diferencia estadísticamente significativa en los estudiantes 
de universidad pública en el grupo sin violencia $(\mathrm{p}=0.007)$ en la conducta de salud organización del sueño (176.27) en comparación con quienes sí la reciben (148.21). De otro lado, en los de universidad privada, se observa diferencia estadísticamente significativa $(\mathrm{p}=0.024)$ en la conducta de salud organización del descanso (97.24) y $(\mathrm{p}=0.001)$ autocuidado y cuidado médico (101.16) en el grupo sin violencia intrafamiliar en contraste con quienes sí existe violencia (80.08) y (75.84) respectivamente. Ello nos permite manifestar que la existencia de violencia interfiere las conductas de salud, básicas como actividad reparadora del organismo, especialmente en el joven, tanto en su organización del sueño, así como en la organización del tiempo libre y el autocuidado. Al revisar ambos grupos se aprecia mayores rangos medios en los estudiantes de ambas universidades cuando no existe desavenencia familiar.

Tabla 6

Comparación de las conductas de salud según violencia intrafamiliar en universidades públicas y privadas

\begin{tabular}{|c|c|c|c|c|c|c|c|c|}
\hline \multirow[b]{2}{*}{ Conductas de salud } & \multicolumn{4}{|c|}{ Pública } & \multicolumn{4}{|c|}{ Privada } \\
\hline & $\begin{array}{c}\text { Ude } \\
\text { Mann- } \\
\text { Whitney }\end{array}$ & $\begin{array}{c}\text { Rango } \\
\text { promedio } \\
\mathrm{C} / \mathrm{VIF} \\
(\mathrm{N}=275)\end{array}$ & $\begin{array}{c}\text { Rango } \\
\text { promedio } \\
\mathrm{S} / \mathrm{VIF} \\
(\mathrm{N}=220)\end{array}$ & $\mathrm{p}$ & $\begin{array}{c}\text { U de } \\
\text { Mann- } \\
\text { Whitney }\end{array}$ & $\begin{array}{c}\text { Rango } \\
\text { promedio } \\
\mathrm{C} / \mathrm{VIF} \\
(\mathrm{N}=275)\end{array}$ & $\begin{array}{c}\text { Rango } \\
\text { promedio } \\
\mathrm{S} / \mathrm{VIF} \\
(\mathrm{N}=220)\end{array}$ & $\mathrm{p}$ \\
\hline $\begin{array}{l}\text { Actividad física y } \\
\text { deporte }\end{array}$ & 11613.500 & 162.38 & 155.23 & .491 & 3690.500 & 86.42 & 91.39 & .516 \\
\hline $\begin{array}{l}\text { Organización del } \\
\text { descanso }\end{array}$ & 11589.000 & 156.49 & 163.96 & .471 & 3152.000 & 80.08 & 97.24 & .024 \\
\hline $\begin{array}{l}\text { Autocuidado y } \\
\text { cuidado médico }\end{array}$ & 11867.500 & 157.96 & 161.79 & .714 & 2791.000 & 75.84 & 101.16 & .001 \\
\hline Hábitos alimentarios & 11596.000 & 162.47 & 155.09 & .481 & 3477.500 & 94.09 & 84.30 & .203 \\
\hline $\begin{array}{l}\text { Consumo de } \\
\text { alcohol, tabaco y } \\
\text { otras drogas }\end{array}$ & 12010.000 & 158.71 & 160.67 & .838 & 3841.500 & 89.81 & 88.26 & .836 \\
\hline $\begin{array}{l}\text { Organización del } \\
\text { sueño }\end{array}$ & 10014.000 & 148.21 & 176.27 & .007 & 3729.000 & 86.87 & 90.97 & .591 \\
\hline
\end{tabular}

5. Comparar los factores de las razones para viviry las dimensiones de la conducta de salud en estudiantes universitarios con y sin violencia intrafamiliar, según género de la ciudad de Lima

Se observa que en los hombres no se encuentran diferencias significativas en uno y otro grupo, con y sin violencia intrafamiliar, ni en las conductas de salud ni en las razones para vivir. Con respecto a las mujeres, del grupo sin violencia, existe diferencia significativa en la conducta de salud organización de descanso o tiempo libre $(155.70 ; p=0.000)$, en la conducta de autocuidado y cuidado médico (148.35; $\mathrm{p}=0.017)$ y en la conducta organización del sueño (146.95; $\mathrm{p}=0.034)$, no así quienes presentan violencia intrafamiliar. 
De otro lado, al analizar las razones para vivir se observa diferencia significativa en las mujeres sin violencia, en la razón para vivir relaciones entre pares (148.66; $\mathrm{p}=0.015)$ y en la razón para vivir de relaciones familiares (158.81; $\mathrm{p}=0.000)$, en comparación al grupo con violencia. Las conductas de salud y las razones para vivir como factor de protección implican un entorno familiar sin violencia; ratificando que la violencia intrafamiliar es un factor de riesgo, así también cobran importancia pares como soporte social.

\section{Interpretación de los datos o discusión}

Una etapa de la vida trascendental viene a ser la juventud en especial lo concerniente a la asunción de comportamientos vinculados con la salud. En el Perú es alarmante y preocupante el detrimento de los hábitos de salud en los jóvenes peruanos, es así que los estudios de población de esta etapa de vida registran un incremento en el número de fallecidos por agresiones, accidentes de tránsito, consumo de alcohol, consumo de drogas, embarazos no deseados, casos con VIH/ SIDA (Calle, 2010; Ministerio de Salud). Pese a ello, los jóvenes no reconocen estos riesgos en forma realista pues no se identifican o lo vinculan muy poco con problemas de salud que suelen aparecer cuando se es adulto (Santrock, 2007; Taylor, 2007). Así la adopción de hábitos no saludables conlleva a problemas de salud y afectan la esperanza de vida, según el Ministerio de Salud (2007). Ello se agrava cuando se asocia vivir dentro de familias donde existe violencia intrafamiliar, lo cual puede precipitar respuestas de estrés que se manifiestan en el inicio de una enfermedad o exacerbar la enfermedad crónica trayendo como consecuencia además que se construya un sistema de creencias que influyen en el modo de evaluar las cosas y tomar decisiones, en sus actitudes y en la forma de vincularse con los demás, sobre los que se van a sustentar las razones para vivir. Lo cual hace relevante estudiar ambos factores, los de riesgo y protectores.

Conforme con Varela, Ochoa y Tovar (2016), las prácticas menos saludables que presentaron los estudiantes universitarios de Bogotá fue la conducta actividad física, revelando un porcentaje alto de jóvenes inactivos; igualmente Becerra (2016) halla en estudiantes de una universidad privada poca o ninguna actividad o ejercicio físico; tales hallazgos son contrarios a lo que se encuentra en la muestra general estudiada pues los universitarios suelen darle importancia a la actividad física la cual se relaciona con la razón para vivir autoevaluación positiva y las interacciones que mantiene con sus pares.

Estudios antecedentes relacionan el ambiente universitario con hábitos de autocuidado y salud e informan una correlación entre rendimiento académico y una salud con características positivas (Feldman, Concalves, Chacón-Puignau, Zaragoza, Bagés, \& Pablo, 2008; Martín, 2007. Citados en Chau y Saravia, 2014), lo cual pone de relieve los estilos de vida saludable sobre la salud, pasando a ser un factor protector cuando incluye actividad física, alimentación balanceada y 
organización del sueño, los que tienen preeminencia pues disminuyen el estrés ligados al desempeño académico en los jóvenes universitarios, generándoles buena salud física y mental (Lema, et al; 2009).

Con respecto al área de recreación y tiempo libre Becerra (2016), encuentra que los universitarios lo utilizan para tener descansos en sus actividades diarias, compartir con su familia y amigos, aunque el cincuenta por ciento de su tiempo libre lo absorben para actividades académicas; de la misma manera encontramos que aquellos estudiantes que presentan como conducta de salud organización del descanso y tiempo libre tienden a presentar como afirmaciones para vivir las expectativas futuras, autoevaluación positiva, creencias de afrontamiento, relaciones con pares y su familia. Otra conducta de salud importante que se encuentra en el presente estudio es que aquellos estudiantes que presentan baja ingesta de alcohol y otras drogas tienden a tener como importante razón para vivir sus relaciones familiares; dato que se visualiza de manera no saludable en aquellos estudiantes universitarios que presentan altos consumos de alcohol asociadas creencias o esquemas disfuncionales tempranos como insuficiente autocontrol, búsqueda de aprobación/ reconocimiento, privación emocional y grandiosidad/ autorización (Huerta, et al.; 2017). Al respecto también Becerra (2016), señala la presencia de consumo de alcohol en los universitarios tanto en hombres como en mujeres; agrega además en lo referente a la organización del sueño que solo un $41.9 \%$ respeta sus horas de sueño, duermen menos de siete horas diarias, como consecuencia presentan somnolencia; en el presente estudio la organización del sueño es tomado muy en cuenta en la muestra general, relacionándose con razones para vivir vinculadas a sus expectativas futuras, adecuadas creencias de afrontamiento, una autoevaluación positiva y buenas relaciones familiares.

De acuerdo a Morales, et al. (2016), entre los factores que conllevan a conducta suicida, es el estar insertos en ambientes familiares con discordia, hostilidad y violencia. Asimismo el tener relaciones interpersonales que protejan y contar con personas significativas actúan como factores de protección contra la depresión y suicidio; tal como también lo refrendan Echávarri, et al. (2015) y García, et al (2007) quienes señalan la existencia de una asociación entre el incremento de razones para vivir y un mínimo riego para la conducta suicida; entre las razones está sentir seguridad en uno mismo, tener destrezas para afrontar la crisis y el desasosiego por la familia. Es así que el vivir dentro de un ambiente familiar sin violencia disminuye los factores de riesgo (Huerta, et al. 2015), debido a que tener conducta de salud y salud mental está influenciada por el entorno social y especialmente el familiar. En el presente estudios se observa en la muestra total que existe asociación en el grupo con violencia familiar, entre la conducta de salud actividad física y deporte con las razones para vivir perspectivas futuras, creencias de afrontamiento, auto evaluación positiva e interacciones entre pares, al parecer esta conducta de salud sería usada como forma de destacar el esfuerzo 
personal lo que les ha conllevado a elaborar razones para vivir. En el caso de la conducta de salud organización del descanso y tiempo libre se asocia, tanto en el grupo con y sin violencia intrafamiliar con los cinco factores de razones para vivir lo que refleja no ser discriminante para encontrar razones para vivir. El autocuidado y cuidado médico es mayor en el grupo sin violencia sobre todo para razones vinculadas con la autoevaluación positiva y relación con los pares siendo dos factores importantes lo personal e interpersonal asociados. En cuanto a los hábitos alimentarios, la razón para vivir que disminuye es relaciones familiares lo que nos indica la relevancia que tiene la familia para tener esta conducta de salud. El bajo consumo de alcohol, tabaco y otras drogas se asocia en el grupo sin violencia en cuatro de los cinco factores de razones para vivir lo cual pasa a ser un factor protector, caso contrario ocurre con el grupo con violencia que se asocia con relación con los pares, lo que nos indicaría que la existencia de violencia al interior de la familia conllevaría a darle más importancia a los compañeros. Por otro lado existe una mayor distorsión en la organización del sueño en el grupo con violencia. Todo lo cual permite afirmar que las conductas de salud influyen en razones para vivir como prevención del suicidio.

Al analizar la correlación entre conductas de salud y razones para vivir según universidad de procedencia pública y privada, encontramos que en la universidad pública existe asociación, en el grupo con violencia, entre las conductas de salud actividad física y deporte, y la organización del descanso con las razones expectativas futuras, autoevaluación positiva, creencias de afrontamiento, relaciones entre pares y familiares; conductas que pueden ser utilizadas como forma de amortiguar la situación familiar en la que están insertos, lo cual propicia factores protectores para la prevención del suicidio pues consideran que sus planes y metas se cumplirán, sienten satisfacción sobre su propia forma de ser y que tienen alternativas para enfrentar la adversidad; que se contrasta con lo manifestado por Hederich \& Camargo (2001; citado en Gantiva; Bello; Vanegas y Sastoque; 2010) quienes afirman que la vivencia de violencia intrafamiliar, precipita respuestas de estrés que alteran la enfermedad crónica o dan lugar a que esta brote y conlleva a la construcción de creencias que influyen en la persona en su manera de actuar, pensar y tomar decisiones. De la misma manera en estos estudiantes, los resultados se pueden considerar como indicadores de comportamiento resiliente como lo señala Huerta (2016) quién encuentra una concordancia positiva y significativa entre el comportamiento resiliente con la ansiedad rasgo en adolescentes de instituciones educativas públicas. En el caso de las privadas, la conducta de salud organización del descanso y tiempo libre es la que presenta asociación con las razones para vivir, tanto en el grupo con y sin violencia, expectativas futuras, autoevaluación positiva y relaciones entre pares, lo cual nos indica una menor diferenciación, en este grupo, respecto a la situación que viven dentro de su ambiente familiar. Por otro lado la conducta, organización del descanso y tiempo libre presenta asociación positiva en el grupo sin violencia, tanto en los de universidad pública como privada, con 
la razón para vivir relaciones familiares; lo cual coincide con Lema, et.al. (2009) quién señala que las conductas de salud están en relación con la madurez de la persona y sus habilidades y competencias, así como la construcción de ambientes de interrelación, por lo que no se concibe que el desarrollo de conductas saludables se encuentre fuera del entorno familiar.

En lo referente al autocuidado y cuidado médico, esta es mayor en los de universidad privada, sin violencia, asociadas a la razón para vivir relaciones entre pares, son los compañeros y amigos los que la propician. En el caso de los de universidad pública ésta conducta tiene relación con autoevaluación positiva, tanto en el grupo con y sin violencia, es decir que para estos universitarios independientemente de su situación familiar esta conducta pasa a ser un factor protector. Con respecto a los hábitos alimentarios en los de universidad pública predomina la autoevaluación positiva en el grupo sin violencia, considerándola importante para su salud; en el con violencia se vincula con las relaciones familiares, es decir que una alimentación saludable estará mediada por la calidad de la relación familiar.

La conducta consumo de alcohol, tabaco y otras drogas, en el grupo de universidad privada sin violencia está vinculada con la razón para vivir relaciones familiares, teniendo la familia un alto significado en lo personal y social. En el caso de los de universidad pública se asocia, en el grupo sin violencia con la razón para vivir creencias de afrontamiento pues consideran que existen varias alternativas para enfrentar las situaciones difíciles; así Koven (2001, citado en Cassaretto y Martinez, 2012), manifestó que se hallan niveles más altos de creencias en el afrontamiento saludable para enfrentar situaciones adversas durante la adultez temprana y tardía, las cuales descienden en la adultez media. Asimismo en el grupo con violencia de universidad pública, la conducta organización del sueño presenta relación con las expectativas futuras y creencias de afrontamiento y en los sin violencia se asocia con autoevaluación positiva es decir que esta conducta en ambientes familiares sin desavenencia familiar conduce a desarrollarla y a generar que se puede afrontar situaciones problemas que acurran en los diferentes contextos.

Cuando se compara según universidad pública y privada, las razones para vivir se encuentra que en ambos grupos ésta es significativa en los sin violencia intrafamiliar, en relaciones familiares, lo cual relieva el papel de la familia. Ello coincide con Vargas y Saavedra (2012), en sus estudios de metaanálisis, encontrando correlación significativa entre el hecho suicida y comunicación familiar, es decir buena interrelación familiar así como considerarse entendido por los integrantes de la familia se vinculó con menos pensamientos e ideación suicida en jóvenes; agregan además que diversos estudios sugieren una relación entre sucesos suicidas y desavenencia familiar, y que la falta de soporte parental están directamente asociados al suicidio. Así un factor protector relevante viene 
a ser la familia pero, a vista de los resultados obtenidos puede transformarse en factor de riesgo si emerge la discordia y violencia dentro de ella.

Al analizar las diferencias en las dimensiones de la conducta de salud según universidad pública y privada se observan diferencias significativas en organización del descanso y autocuidado y cuidado médico en los de universidad privada sin violencia. Al mismo tiempo se aprecia en los estudiantes de universidad pública, sin violencia que la conducta de salud que más destaca es la de organización del sueño. Ello permite afirmar que la pertenencia a una familia sin violencia es un factor protector hecho que Gantiva; Bello; Vanegas y Sastoque (2010) destacan que las variaciones en el ciclo familiar pueden incitar irritación emocional, inestabilidad y desestabilizar la salud.

Finalmente cuando se revisan las diferencias según sexo se encuentra que son las mujeres, del grupo sin violencia, las que presentan conductas de salud (organización del descanso, autocuidado y cuidado médico y organización del sueño) y razones para vivir (relaciones entre pares y relaciones familiares) no así en los hombres. Lo cual no se observa cuando hay violencia intrafamiliar. De manera contraria halla Becerra (2016), pues encuentra que los hombres presentan elevados conductas de salud en el área de actividad física y deporte y que en el caso de las mujeres es en su bajo consumo de alcohol, tabaco y otras drogas. Asimismo Vargas y Saavedra (2012) reportan que la prevalencia de vida de ideaciones e intentos suicidas fue concluyentemente más alto en mujeres al contrastarlos con varones en 11 estudios, lo cual también Huerta, et.al. (2015) halla que en las mujeres hay un predominio de indicadores de depresión (15.46) y en entornos de violencia intrafamiliar los niveles de depresión se acrecientan en las mujeres (16.4) en contraste con los hombres. De esta manera podemos afirmar que el contexto familiar es fundamental para el desarrollo de conductas de salud y razones para vivir, pasando a ser este, tanto un factor protector como de riesgo para la prevención de fenómenos suicidas y el desequilibrio del estado de salud.

\section{CONCLUSIONES}

- En la muestra general existe relación positiva y significativa entre las dimensiones de las conductas de salud con las razones para vivir en especial la actividad física y deporte y el autocuidado y cuidado médico con autoevaluación positiva y las relaciones que mantiene con sus pares, lo cual relieva la vinculación entre salud física y salud mental.

- En la muestra en general, la conducta organización del descanso y tiempo libre se asocia con las razones para vivir, perspectivas futuras, creencias de afrontamiento, autoevaluación positiva, relaciones con pares y su familia. Así mismo los que presentan bajo consumo de alcohol tienen como razón para vivir la relación con su familia. 
- La conducta de salud y la salud mental está influenciada por el entorno social, especialmente el familiar. Cuando existe violencia intrafamiliar hay asociación entre las conductas de salud actividad física y deporte y organización del sueño con las razones expectativas futuras, creencias de afrontamiento, autoevaluación positiva y relaciones entre pares. El bajo consumo de alcohol se asocia a cuatro factores de las razones para vivir, pasando a ser un factor protector para la prevención del suicidio.

- En los estudiantes de universidad pública, con violencia intrafamiliar, se relacionan las conductas de salud actividad física y deporte, y la organización del descanso con las razones expectativas futuras, creencias de afrontamiento, autoevaluación positiva, relaciones entre pares y familiares. Conductas que se constituyen en factores protectores para la prevención del suicidio e indicadores de comportamiento resiliente.

- En los de universidad privada no se aprecia mayor diferenciación tanto cuando existe o no violencia intrafamiliar en cinco de las dimensiones de la conducta de salud; a excepción de autocuidado y cuidado médico con relaciones entre pares cuando no existe violencia.

- En los estudiantes de ambas universidades, cuando no existe violencia, las conductas de organización del descanso, y el bajo consumo de alcohol, tabaco y otras drogas se asocian, con la razón para vivir relaciones familiares. La familia tienen un alto significado en lo personal y social.

- La variable razones para vivir, tanto en los estudiantes de universidad pública como privada es significativa cuando no existe violencia en relaciones familiares.

- En los estudiantes de universidad privada existen diferencias significativas, en el grupo sin violencia, en las conductas organización del descanso y tiempo libre, autocuidado y cuidado médico y la organización del sueño. Cuando no existe violencia, en los de universidad pública es la organización del sueño la más importante.

- Las mujeres, sin violencia, presentan conductas de salud (organización del descanso, autocuidado y cuidado médico y organización del sueño) y razones para vivir (relaciones entre pares y relaciones familiares) no así en los hombres. Lo cual no se observa cuando hay violencia intrafamiliar en ambos géneros.

- Las conductas de salud en ambientes familiares sin violencia intrafamiliar conducen a fortalecer los factores protectores propiciando razones para vivir para la prevención de fenómenos suicidas y descompensación del estado de salud. 


\section{REFERENCIAS}

Becerra, S. (2016). Descripción de las conductas de salud en un grupo de estudiantes universitarios de Lima. Revista de Psicología 34(2) 239-260. Recuperado en http:// dx.doi.org/10.18800/psico.201602.001

Calle, M. (2010). Análisis de la Situación de salud de los jóvenes peruanos - Ministerio de Salud, Lima-Perú. ftp://ftp2.minsa.gob.pe/descargas/dgsp/../ASIS\%20Joven\%20 30\%2004\%202013.pdf

Cassaretto, M y Martínez, P. (2012). Razones para vivir en jóvenes adultos: validación del RFL-YA. Revista de Psicología, 30(1), 169-188.

Chau, C. y Saravia, J. (2014). Conductas de Salud en Estudiantes Universitarios Limeños: Validación del CEVJU. Revista Iberoamericana de Diagnóstico y Evaluación, 41 (1), $90-103$.

Corsi, J. (1997). Violencia Familiar. Una mirada interdisciplinaria sobre un grave problema social. Buenos Aires, Argentina: Paidos

Echávarri, O., Morales, S., Bedregal, P., Barros, J., Maino, M.,Fischmanb (2015). ¿Por qué no me suicidaría? Comparación entre pacientes hospitalizados en un servicio de psiquiatría con distinta conducta suicida. Psykhe, 24, 1---11.

Gantiva, C; Bello, J; Vanegas, E y Sastoque, Y. (2010) "Relación entre el consumo excesivo de alcohol y esquemas maladaptativos tempranos en estudiantes universitarios". Revista Colombiana de Psiquiatría, 39, 362-374.

García, J., Palacio, C., Arias, S., Ocampo, M., Calle, J. y Restrepo, D. (2007). Características asociadas al riesgo de suicidio valorado clínicamente en personas con intento reciente. Revista Colombiana de Psiquiatría, 36(4), 610---627.

Hernández, R.; Fernández, C. y Baptista, P. (2010). Metodología de la Investigación. México: Editorial Mc Graw Hill.

Huerta, R., Aliaga, J., Campos, E., Ramirez, N., Santivañez, R., Ramos, J., Murillo, L., Falcón, C., Treneman, N., Tejada, M., Castro, M. y Pérez, P. (2015) Depresión y cólera-hostilidad en alumnos víctimas de violencia intrafamiliar, de cuarto grado de secundaria de instituciones educativas estatales de zonas urbano marginal de la ciudad de Lima. Revista de Investigación en Psicología. Facultad de Psicología. UNMSM. 18(2), pp. 83-100.

Huerta, R. (2016). Relación entre el tipo de familia y la ansiedad con el comportamiento resiliente es adolescentes en situación de pobreza (Tesis para optar el Grado de Doctor en Psicología). Universidad Nacional Mayor de San Marcos, Lima-Perú.

Huerta, R.; Miljanovich, M.; Aliaga, J.; Campos, E.; Ramírez, N.; Delgado, E.; Ramos, J. y Murillo, L. (2017). Esquemas disfuncionales tempranos y consumo de alcohol en estudiantes universitarios, según carrera profesional de una universidad pública de la ciudad de Lima. Revista de Investigación en Psicología. 20(2), pp. 309 - 326 ISSN L: 1560 - 909X Facultad de Psicología UNMSM. DOI: http://dx.doi.org/10.15381/rinvp. v20i2.14043 
Razones para vivir y conducta de salud en estudiantes universitarios con y sin violencia intrafamiliar, según procedencia (pública y privada) de la ciudad de Lima

Lema, L.; Salazar, I.; Varela, M.; Tamayo, J.; Rubio, A. y Botero, A. (2009). Comportamiento y salud de los jóvenes universitarios: satisfacción con el estilo de vida. Pens Psicol 5(12): 71-88.

Linehan, M. M., Goodstein, J. L., Nielsen, S. L. y Chiles, J. A. (1983). Reasons for staying alive when you are thinking of killing yourself: The Reasons for Living Inventory. Journal of Consulting and Clinical Psychology, 51(2), 276-286.

Ministerio de Salud- MINSA (2007). Norma Técnica para la Atención Integral de Salud en la etapa de Vida Adolescente. http://www.minsa.gob.pe/portal/servicios/ susaludesprimero/adolescente/adol-doc.asp Documento técnico promoviendo universidades saludables. Lima: Ministerio de Salud.

Morales, S.; Fischmanb, R.; Echavarri, O.; Barros J.; Arijo, I; Moya. C.; De la Paz, M. y Nuñez, C. (2016). Vivencia-expresión de la rabia y razones para vivir en un grupo de pacientes chilenos con riesgo suicida. Revista Iberoamericana de Psicología y Salud. 7, 60-68. Recuperado en http://dx.doi.org/10.1016/j.rips.2016.03.002

Organización Mundial de la Salud. (2001). World Health Report 2001. Mental health: New understanding, new hope. Ginebra, Suiza

Organización Mundial de la Salud. (2018). OMS. Suicidio. Centro de Prensa. www.who. int/mediacentre/factsheets/fs398/es/ Ginebra, Suiza.

Osman, A., Downs, W., Kopper, B., Barrios, F., Baker, M. y Osman, J. (1998). The Reasons for Living Inventory for Adolescents (RFL-A): Development and psychometric properties. Journal of Clinical Psychology, 54(8), 1063-1078.

Paredes, C., Orbegoso, O. \& Rosales, P. (2006). Caracterización de pacientes con intento de suicidio en un hospital general de Lima, 1995-2004. Revista Peruana de Medicina Experimental y Salud Pública, 23(4), 293-296.

Rosero, M. (2008). Propiedades psicométricas de una versión adaptada y una ampliada del Inventario de Razones para Vivir en Adultos Jóvenes (RFL-YA, Gutiérrez et al., 2002), en una muestra de estudiantes universitarios de la ciudad de San Juan de Pasto (Tesis de licenciatura inédita). Universidad de Nariño, Colombia.

Salazar, I., Varela, M.T., Lema, L.F., Tamayo, J.A. y Duarte, C. (2010). Manual del Cuestionario de Estilos de Vida en jóvenes universitarios (CEVJU-R2). Pontificia Universidad Javeriana Cali.

Salazar, I., Varela, M.T., Lema, L.F., Tamayo, J.A. y Duarte, C. (2010). Evaluación de las conductas de salud en jóvenes universitarios .Revista Salud Pública. 12 (4): 599-611.

Santrock, J. (2006). Psicología del desarrollo: El ciclo vital. Décima edición. Madrid, España: McGraw-Hill.

Taylor, S. (2007). Psicología de la salud. México D.F.: McGraw Hill.

Varela, M.; Ochoa, A y Tovar, J. (2016). Tipologías de estilos de vida en jóvenes universitarios. Rev. Univ. Salud 18(2): 246-256. Recuperado en www.scielo.org.co/ $\mathrm{pdf} / \mathrm{reus} / \mathrm{v} 18 \mathrm{n} 2 / \mathrm{v} 18 \mathrm{n} 2 \mathrm{a} 06$ 
Vargas, H. y Saavedra, J. (2012). Factores asociados con la conducta suicida en adolescentes. Revista Neuropsiquiatría 75 (1), 19-28. Recuperado en www.upch.edu. pe/vrinve/dugic/revistas/index.php/RNP/article/viewFile/.../1567

Vargas, H., Tovar, H. y Valverde, J. (2010) Prevalencia y factores asociados con el episodio depresivo en adolescentes de Lima Metropolitana y Callao. Revista Peruana de Epidemiología. Vol.14(2).

Wang, M., Lightsey, O., Pietruszka, T., Uruk, A. \& Wells, A. (2007). Purpose in life and reasons for living as mediators of the relationship between stress, coping, and suicidal behavior. The Journal of Positive Psychology, 2(3), 195-204. 